\title{
Detection Of Twisted Blade In Multi Stage Rotor System
}

\author{
W.K. Ngui ${ }^{1,}$,, M. Salman Leong ${ }^{2}$, L.M. Hee ${ }^{3}$ and Ahmed. M. Abdelrhman ${ }^{4}$ \\ 1,2,3,4 Institute of Noise \& Vibration, Universiti Teknologi Malaysia Kuala Lumpur, Jalan Semarak, \\ 54100 Kuala Lumpur, Malaysia \\ awaikeng84@yahoo.com
}

Keywords: twisted blade, multi stage rotor system

\begin{abstract}
This paper studies the detection of twisted blade in a multi stages rotor system. Experimental study was undertaken to simulate twisted blade conditions in a three stages rotor system. The feasibility of vibration analysis as the technique to detect twisted blade based on the rotor operating frequency and its blade passing frequency was investigated in this study. Experimental results show that twisted blade can be easily detected by looking into the pattern of the vibration spectrum and its individual peaks.
\end{abstract}

\section{Introduction}

Lately, there is an increased of interest in blade faults diagnosis based on the publications in the field. There are several studies in the open literature reports on the characteristics and technique used to detect and diagnose various type of blade faults. Blade faults such as blade rubbing, blade fatigue failure, blade deformation, blade fouling and loose blade are commonly simulated and studied by researcher. In the past, researcher have applied various type of techniques to detect and diagnose blade faults such as vibration analysis, pressure analysis, temperature analysis, acoustic analysis and others. A review of blade fault diagnosis can be found in [1].

Vibration analysis is the most widely used technique for blade faults detection and diagnosis. Previous studies done by Simmons [2,3] and Parge et al. [4] have discovered that the relative change in blade passing frequency (BPF) and its harmonic amplitude could be used for blade faults detection. Lim et al. [5] have proposed the vibration response approach for blade rubbing detection in a single stage rotor system. They also commented that severity of rubbing could be estimated based on the magnitude and pattern of the vibration spectrum. Lim and M.S. Leong [6] extended his work to identify the position of the faulty blade in rotor. They have proposed to suppress the harmonic of operating speed in order to reduce the influence of these frequency components from affecting the results for blade fault diagnosis. However, this is contrary to a study conducted by Loukis et al. [7] as they had filter out all frequencies others than the rotor operating frequency and then to calculate the spectral difference patterns which is used for blade faults detection. Besides that, Ahmed et al. [8,9] studies the feasibility of vibration spectrum and wavelet analysis for multi stages blade fault diagnosis. They showed that vibration spectrum can clearly depict the location and the stage of blade rubbing. The analysis of rotor operating frequency and its blade passing frequency was used for the stage blade rubbing diagnosis.

These literature shows that multi stage blade faults analysis and especially the twisted blade condition has not yet been extensively investigated in the open literatures. The purpose of this study is to investigate method to detect twisted blade in a multi stage rotor system using vibration analysis. 


\section{Experimental study}

An experimental study was conducted to simulate twisted blade condition in a multi stage rotor system. The test rig consists of $8,11,13$ number of blades located at first, second and third row of the rotor respectively. Accelerometers were mounted on the rotor casing in vertical and horizontal position to pick up the vibration signals. A photograph showing the multi stage rotor system is shown in Fig. 1.

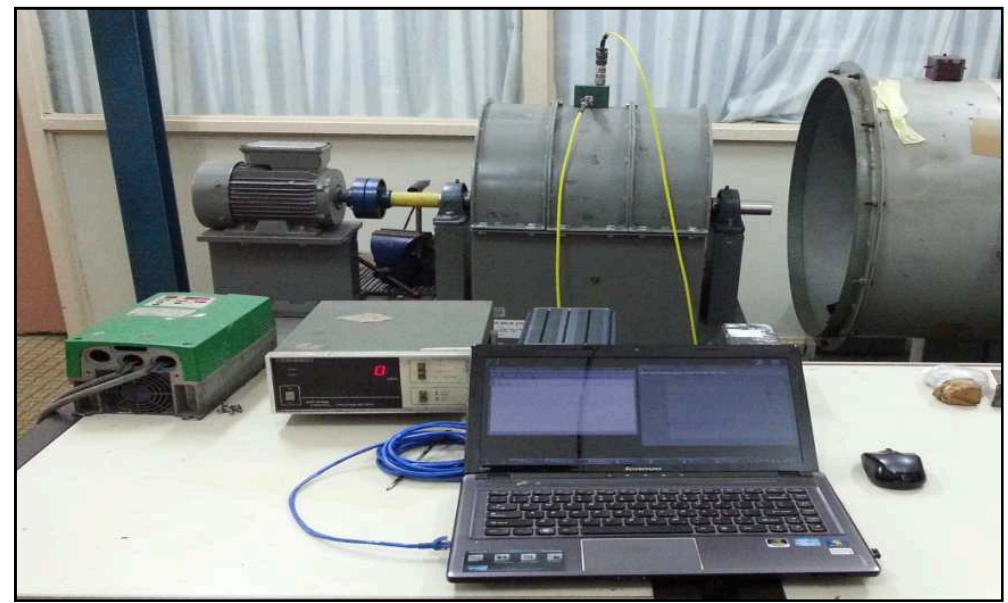

Fig. 1. Multi stage rotor system

In order to study the vibration response of twisted blade, the healthy blade was replaced with a twisted blade. In this study, four condition of twisted blade were simulated, namely, baseline healthy condition, single twisted blade at first row, single blade twisted at second row and single blade twisted at third row. All vibration signals were measures under steady state condition (1200 rpm).

\section{Experimental results and discussion}

As mentioned in the previous section, frequencies others that the rotor operating frequency and its blade passing frequency (BPF) are filtered out intentionally in order for the detection of twisted blade. The rotor operating frequency is $20 \mathrm{~Hz}$, while the blade passing frequencies (BPF) for the first, second and third row of the rotor are $160 \mathrm{~Hz}, 220 \mathrm{~Hz}$, and $260 \mathrm{~Hz}$ respectively.

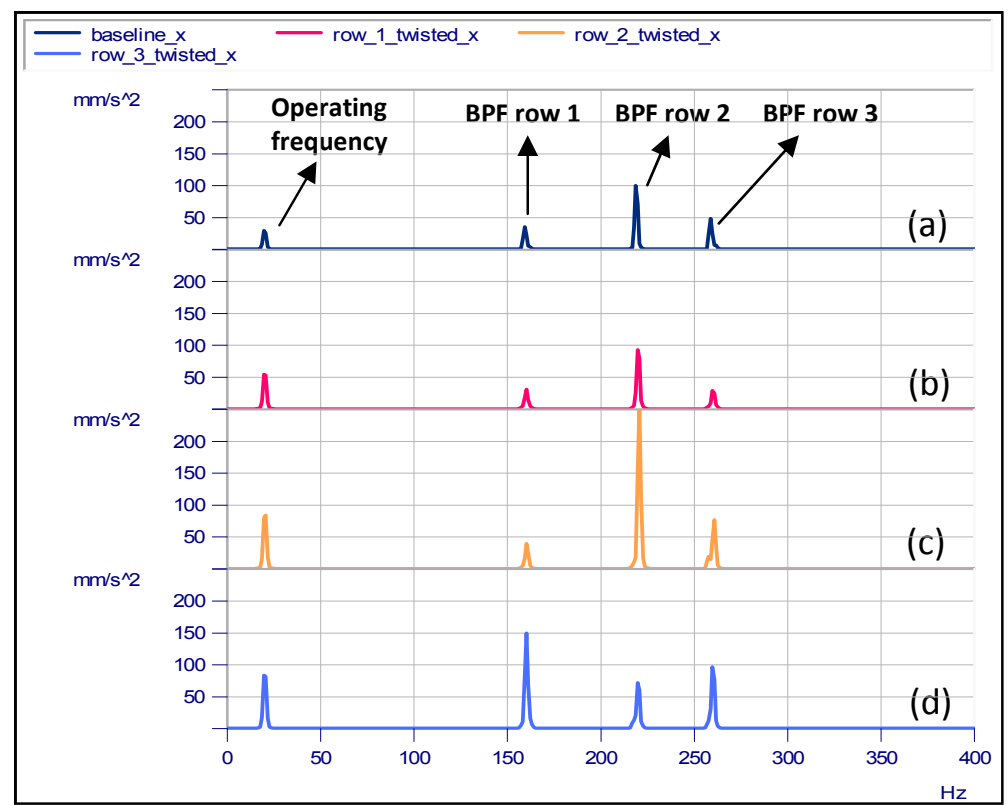

Fig. 2. Vibration spectrum for horizontal direction 


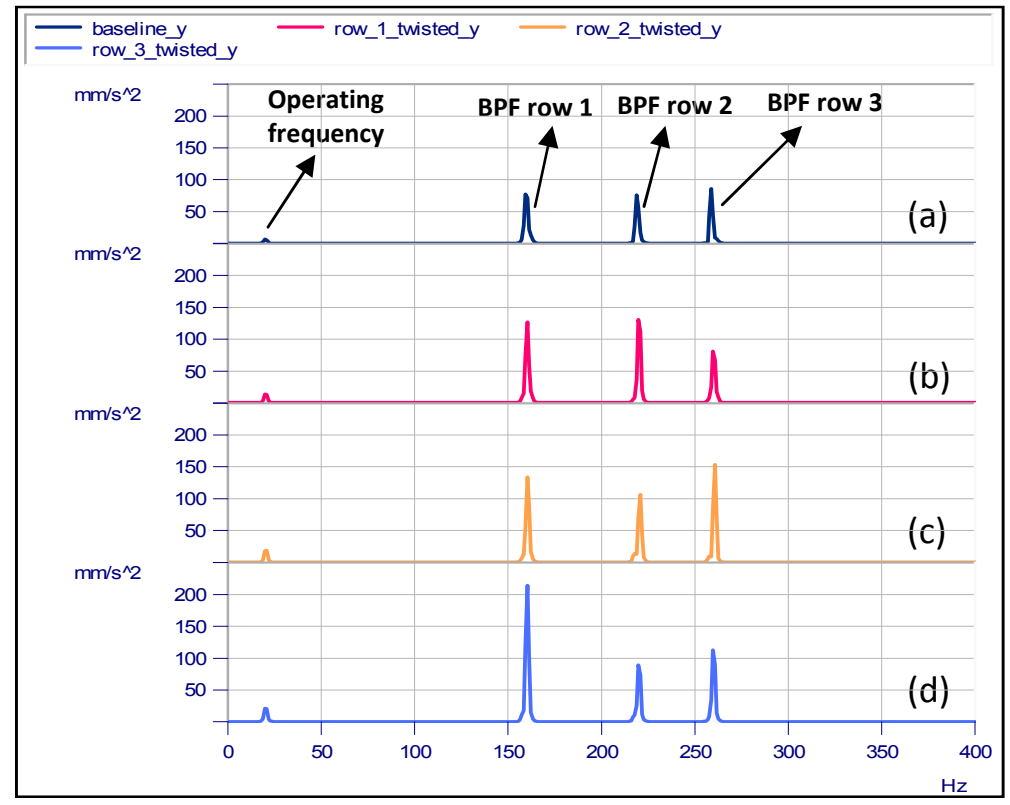

Fig. 3. Vibration spectrum for vertical direction

Fig. 2 and 3 show the vibration spectrum of the rotor casing in horizontal and vertical axes for various twisted blade conditions induced in the experiment. From these figures, we can notice that when twisted blade conditions were induced in the rotor, the amplitude of the rotor operating frequency increases accordingly. By monitoring the vibration amplitude of the individual blade passing frequency, the specific row of which the twisted blade is located can be reasonably identified. The curve that connecting the amplitude of the rotor operating frequency and its blade passing frequency in horizontal and vertical axes for various twisted blade conditions are shown in Fig. 4 and 5. These figure show that difference twisted blade condition will have difference unique curve pattern.

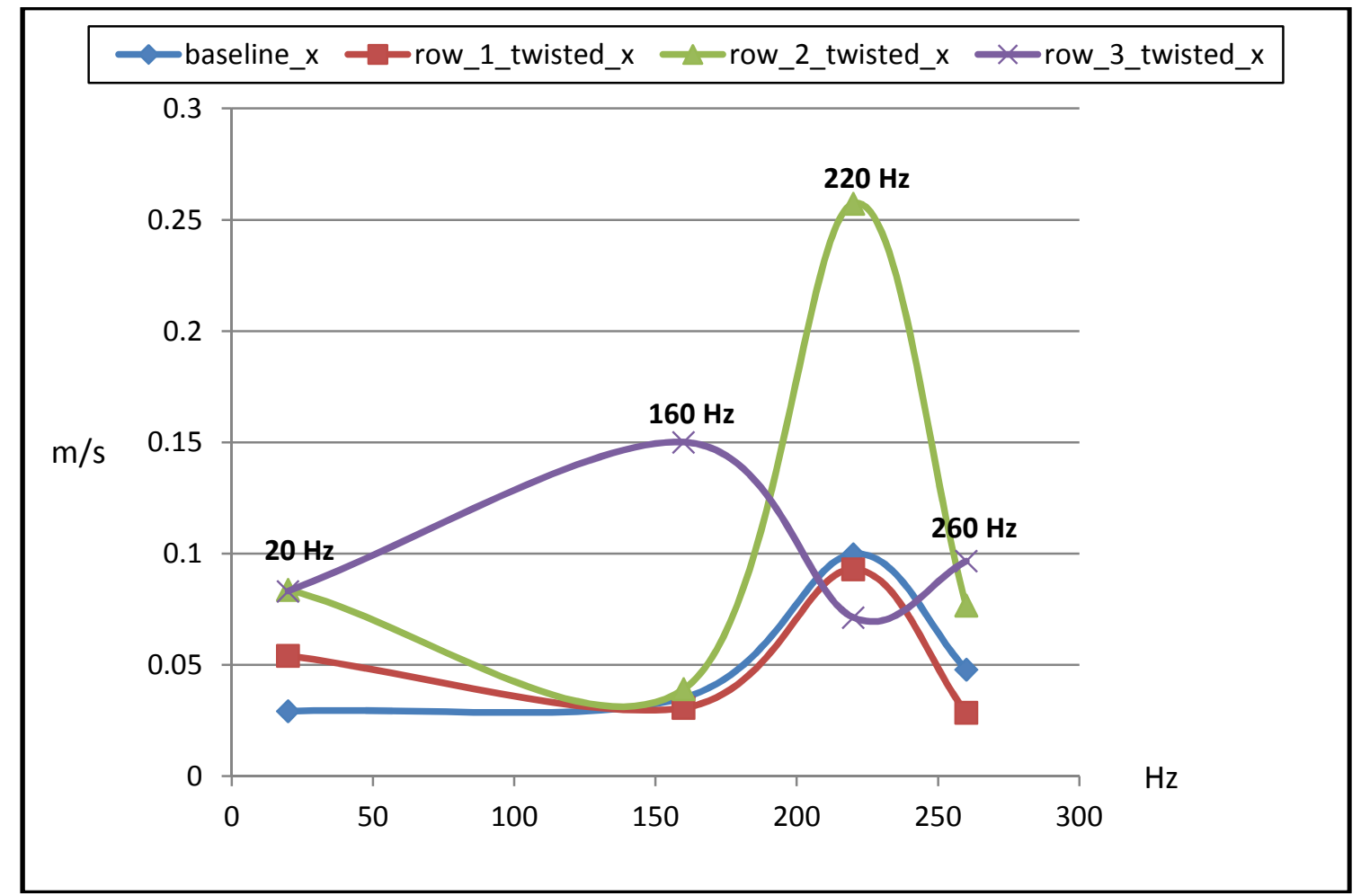

Fig. 4. Curve pattern of the rotor operating frequency and its BPF amplitude in horizontal axis 


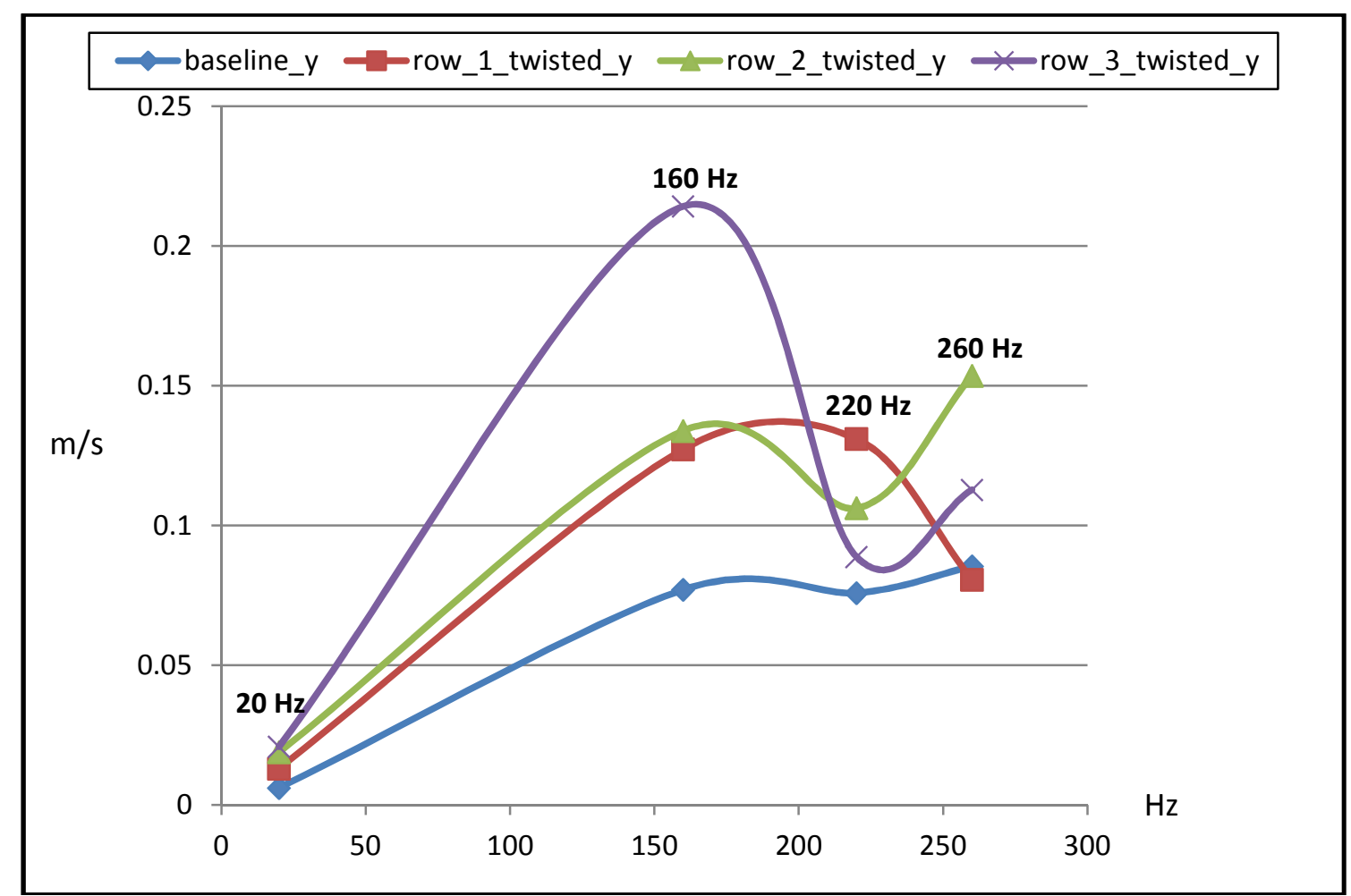

Fig. 5. Curve pattern of the rotor operating frequency and its BPF amplitude in vertical axis

\section{Conclusion}

The objective of this study was to detect twisted blade in multi stage rotor system using vibration spectrum analysis. The experimental results shown that rotor operating frequency and its blade passing frequency are useful for the detection of twisted blade in multi stage rotor system. However, research on twisted blade detection should be focused more on the identification of the exact position of twisted blade in the row, which is still not possible with the current method.

\section{Acknowledgement}

This work is supported by the UTM Flagship Grant (Q.K130000.2409.01G44) and Research University Grant of UTM (Q.K130000.2540.06H51 and Q.J130000.2524.05H01) financed by the Ministry of Education, Malaysia. The main author is joint-funded by the Ministry of Education and Universiti Malaysia Pahang for his PhD study.

\section{References}

[1] A. M. Abdelrhman, L. M. Hee, M. S. Leong, and S. Al-Obaidi, "Condition Monitoring of Blade in Turbomachinery: A Review," Adv. Mech. Eng., vol. 2014, pp. 1-10, 2014.

[2] H. Simmons, "A Non-intrusive Method for Detecting HP Turbine Blade Resonance," in ASME Paper 86 - JPGC - Pwr - 36, 1986.

[3] H. Simmons, "Nonintrusive Detection of Turbine Blade Resonance," in Proceddings of the 3rd EPRI Conference on Incipient Failure Detection in Power Plants, 1987.

[4] P. Parge, B. Trevillion, and P. Carle, "Non-Instrusive Vibration Monitoring for Turbine Blade Reliability," in Proceedings of the 2nd International Machinery Monitoring and Diagnostic Conference, 1990, pp. 435-446. 
[5] L. M. Hee, M. S. Leong, and N. W. Keng, "Vibration Analysis of Rub in Rotating Machinery," Appl. Mech. Mater., vol. 390, pp. 215-219, Aug. 2013.

[6] M. H. Lim and M. S. Leong, "Reconstruction of Vital Blade Signal from Unsteady Casing Vibration," Adv. Mech. Eng., vol. 2014, pp. 1-9, 2014.

[7] E. Loukis, K. Mathioudakis, K. Papailiou, and E. Data, "A Procedure for Automated Gas Turbine Blade Fault Identification Based on Spectral Pattern Analysis,” no. 91, 2014.

[8] A. M. Abdelrhman, M. S. Leong, L. M. Hee, and W. K. Ngui, "Application of Wavelet Analysis in Blade Faults Diagnosis for Multi-Stages Rotor System," Appl. Mech. Mater., vol. 393, pp. 959-964, Sep. 2013.

[9] A. M. Abdelrhman, M. S. Leong, L. M. Hee, and K. H. Hui, "Vibration Analysis of Multi Stages Rotor for Blade Faults Diagnosis," Adv. Mater. Res., vol. 845, pp. 133-137, Dec. 2013. 